STUDI

FRANCESI

\section{Studi Francesi}

Rivista quadrimestrale fondata da Franco Simone

180 (LX | III) | 2016

Varia

\title{
Lettres et musique, l'alchimie fantastique. La musique dans les récits fantastiques du romantisme français (1830-1850), éd. Stéphane Lelièvre
}

\section{Emilie Pézard}

\section{(2) OpenEdition \\ Journals}

Édition électronique

URL : http://journals.openedition.org/studifrancesi/5360

DOI : $10.4000 /$ studifrancesi.5360

ISSN : 2427-5856

Éditeur

Rosenberg \& Sellier

\section{Édition imprimée}

Date de publication : 1 décembre 2016

Pagination : 537-538

ISSN : 0039-2944

\section{Référence électronique}

Emilie Pézard, « Lettres et musique, l'alchimie fantastique. La musique dans les récits fantastiques du romantisme français (1830-1850), éd. Stéphane Lelièvre », Studi Francesi [En ligne], 180 (LX | III) | 2016, mis en ligne le 01 janvier 2017, consulté le 18 septembre 2020. URL : http://journals.openedition.org/ studifrancesi/5360 ; DOI : https://doi.org/10.4000/studifrancesi.5360

Ce document a été généré automatiquement le 18 septembre 2020.

\section{cc) $(9)$}

Studi Francesi è distribuita con Licenza Creative Commons Attribuzione - Non commerciale - Non opere derivate 4.0 Internazionale. 


\title{
Lettres et musique, l'alchimie
} fantastique. La musique dans les récits fantastiques $d u$ romantisme français (1830-1850), éd. Stéphane Lelièvre

\author{
Emilie Pézard
}

\section{RÉFÉRENCE}

Lettres et musique, l'alchimie fantastique. La musique dans les récits fantastiques du romantisme français (1830-1850), textes rassemblés, annotés et présentés par Stéphane LELIÈVRE, Château-Gontier, Éditions Aedam Musicae, 2015, 371 pp.

1 Cette anthologie réunit quatorze récits qui se situent au point de convergence d'un mouvement esthétique, d'un genre et d'une thématique: le lecteur est amené à découvrir ou à relire des récits fantastiques de George Sand, Jules Janin, Théophile Gautier, Samuel-Henry Berthoud, Théophile de Ferrière, Charles Rabou, Frédéric Mab, Raymond Brucker et Alexandre Dumas, parus pendant l'époque romantique, et qui accordent tous une place centrale à la musique.

2 Le choix de se limiter au domaine français et à des bornes chronologiques assez étroites permet d'inclure dans le volume des textes rares ou méconnus. L'intérêt d'une anthologie est aussi de rendre accessibles pour le lecteur contemporain des textes qui ne seraient pas publiés de façon isolée: on sait donc gré à Stéphane Lelièvre d'avoir classé «au chapitre des regrets» des récits comme Euphonia de Berlioz ou Gambara de Balzac, déjà édités, pour privilégier des textes moins connus. On regretterait presque la présence de La Femme au collier de velours, de Dumas, qui occupe une centaine de pages dans cet ouvrage qui en compte 350, à la place duquel on aurait été bien curieux de lire cet «atypique Chien des musiciens de Champfleury» qui est évoqué dans l'introduction. Il faut cependant saluer le choix qu'a fait Stéphane Lelièvre de proposer les textes tels 
qu'ils ont été publiés pour la première fois, le plus souvent dans des revues: même les récits plus connus du lecteur présentent ainsi un intérêt nouveau, et l'anthologie peut vraiment donner à lire ce qu'était «le récit fantastico-musical» dans les années 1830 et 1840.

L'approche adoptée est en effet résolument historique. S. Lelièvre n'élude pourtant pas la question épineuse de la valeur littéraire et, du point de vue du style, on s'accorde tout à fait avec ses jugements sur «Trois hommes: aventure allemande» de SamuelHenry Berthoud, qui «ne constitue pas le meilleur texte de cette anthologie» (p. 88), ou sur «Tobias Guarnerius» de Charles Rabou, «un texte nettement supérieur à bon nombre d'autres diableries fantastico-musicales de l'époque» (p. 167), qui méritait bien d'être relu indépendamment des Contes bruns où il a d'abord été publié. Mais, au-delà de ces bonnes ou mauvaises surprises sur la qualité de leur écriture, les textes recueillis ont tous le mérite de présenter un aspect spécifique, souvent inattendu, du fantastique à l'époque romantique et des significations que revêtait alors la musique.

4 L'introduction rappelle de façon bienvenue que le fantastique tel qu'on l'entendait dans les années 1830 n'a que peu à voir avec les constructions théoriques de la seconde moitié du $\mathrm{xx}^{\mathrm{e}}$ siècle. Ignorant superbement, et à juste titre, la définition de Tzvetan Todorov pour qui «le fantastique est fondé essentiellement sur une hésitation du lecteur [...] quant à la nature d'un événement étrange», Stéphane Lelièvre présente le fantastique comme «l'irruption de l'étrange dans un univers familier». La formule fait directement écho à la définition que donnait Pierre-Georges Castex dans Le Conte fantastique en France - «une intrusion brutale du mystère dans la vie réelle»-, avec cependant une différence significative, la disparition de la dimension dysphorique, «brutale», de ce heurt de l'imaginaire et du réel. Stéphane Lelièvre rappelle en effet que ce n'est que progressivement, au fil du XIX siècle, que s'est imposée l'idée d'un fantastique intimement lié à l'effroi ou au malaise: à l'époque romantique, le fantastique est encore le jumeau sémantique de la fantaisie, et désigne «le fait de vivre une expérience inédite, faisant éprouver des émotions littéralement surhumaines, qu'elles génèrent un plaisir intense - ou un inquiétant vertige» (p.13). Tout en accordant une place importante aux textes du «fantastique noir», qui occupent deux sections dans la table des matières, Stéphane Lelièvre inclut donc également des récits qu'un lecteur $\mathrm{du} \mathrm{xxI}^{\mathrm{e}}$ siècle pourrait juger «aux frontières du fantastique» (titre de la première section), plus proches du conte merveilleux, comme «Le Nid de rossignols» de Gautier, ou des «récits simplement étranges [...] mettant en scène des personnages curieux, excentriques» (p. 13). L'anthologie donne ainsi à retrouver l'unité complexe de la veine fantastique telle que le romantisme français l'a héritée de Hoffmann, figure tutélaire qui domine tous les récits recueillis.

5 Cette définition élargie du fantastique, plus conforme à l'histoire littéraire, explique l'importance qu'occupe dans ce genre la musique, qui n'occupe pas a priori la même place de choix que les vampires ou le double dans le canon des motifs fantastiques. C'est que la musique, comme le fantastique en 1830, est semblable à cette grande «échelle dressée vers le ciel» décrite par Hoffmann dans les Frères de Saint-Sérapion: la musique est naturellement fantastique, elle en qui on admire «le pouvoir [...] de faire pénétrer l'initié dans un extra-monde, d'essence incontestablement divine». Liée à l'idéal, au sublime, à la révolte contre les limites de la condition humaine, elle donne lieu à de multiples combinaisons narratives où s'exprime la complexité de la sensibilité romantique. 
6 Outre le choix du sujet et des textes, il faut saluer l'exceptionnelle richesse de l'appareil critique, qui fait de cette anthologie non seulement une source primaire cruciale pour l'étude du romantisme, mais aussi un volume d'informations et d'analyses dont la qualité ne cesse d'impressionner le lecteur. Le volume s'ouvre sur une introduction, qui présente les problématiques abordées et offre une interprétation synthétique des récits recueillis, et se termine avec un index des noms, une bibliographie complète et une table des matières. Pour chaque récit, outre un appareil de notes éclairant précisément les références ou le vocabulaire, le lecteur trouve une «notice sur l'auteur» - composée de repères bio-bibliographiques pour les auteurs les moins connus et, pour Gautier, Sand et Dumas, d'une présentation approfondie des rapports de l'écrivain avec la musique et le fantastique -, et une «notice sur le texte», commentaire éclairant qui est parfois aussi long que le récit lui-même. Cette richesse de l'appareil critique, l'intérêt des textes qui sont compilés - tant du point de vue de l'écriture que de l'histoire littéraire ou des représentations de la musique -, les analyses précises et limpides de Stéphane Lelièvre font de cette anthologie un ouvrage que liront avec grand plaisir aussi bien les universitaires que les amateurs cultivés.

7 Notons enfin, pour le louer, le beau travail effectué par la maison d'éditions castrogontiérienne Aedam Musicae: le volume souple, aux larges pages et à l'impression impeccable se caractérise autant par le confort de lecture qu'il offre que par son élégance. À une époque où les bibliothèques numériques rendent accessibles nombre de textes rares, mais dans des versions en ligne souvent pénibles à lire, la précision peut ne pas être inutile. 